\title{
Anatomía aplicada de las fontanelas
}

\author{
J.L. Bustamante; L.A. Miquelini; M. D’Agustini* y A.M. Fontana
}

Cátedra de Anatomía "C". Facultad de Ciencias Médicas. Universidad Nacional de La Plata. La Plata. Buenos Aires. Argentina. *Servicio de Neurocirugía. Hospital de Niños de La Plata "Sor María Ludovica”. La Plata. Buenos Aires. Argentina.

Trabajo preliminar presentado en el XLIV Congreso Argentino de Anatomía de la Asociación Argentina de Anatomía, Noviembre de 2007, La Plata.

\section{Resumen}

Objetivo. Describir la anatomía de las fontanelas y actualizar las aplicaciones del conocimiento de las mismas.

Métodos. Se utilizaron 7 preparados cadavéricos de cabezas fetales, 2 cráneos secos y 2 huesos frontales, se analizaron 48 fontanelas anteriores de pacientes pediátricos con y sin patología asociada, así como también casos quirúrgicos y neuroecografías. Dichas estructuras fueron sometidas a mediciones $\mathbf{y}$ posterior análisis.

Resultado. Se evidenció las medidas, estructura y límites de las fontanelas en las cabezas y cráneos fetales, así como la evaluación de pacientes pediátricos y las medidas de las fontanelas en diferentes patologías.

Conclusión. Debido a sus características anatómicas, las fontanelas son una verdadera ventana al endocráneo, lo que determina sus múltiples aplicaciones clínico-quirúrgicas.

PALABRAS CLAVE: Anatomía. Fontanelas. Neuroecografía. Punciones.

\section{Applied anatomy of the fontanelles}

\section{Summary}

Objective. To make a description of the anatomy of fontanelles and to update what is currently known about their applications.

Method. 7 cadaveric heads, 2 dry skulls and 2 frontal bones of foetus were used, and 48 anterior fontanelles from paediatric patients with or without associated pathology were examined. Surgical cases and neuroso-

Recibido: 6-11-09. Aceptado: 27-12-09 nography were also used. All the structures were measured and subsequently analysed.

Results. The size, structure and limits of the fontanelles were evidenced in the cadaveric head and skulls, as well as the evaluation of paediatric patients and the size of their fontanelles in different pathologies.

Conclusion. Owing to their anatomical characteristics, fontanelles are a true window to the inside of the skull, which determines their multiple clinical and surgical applications.

KEY WORDS: Anatomy. Fontanelles. Neurosonography. Puncture.

\section{Introducción}

Los huesos de la bóveda craneal se originan por el proceso de osificación membranosa, a diferencia de gran parte del organismo, cuya osteogénesis es precedida por un esbozo cartilaginoso ${ }^{22,36}$.

Los puntos de osificación en los huesos de la calota son centrales, y luego se extienden hacia la periferia, lo que implica que al momento del nacimiento, dichos huesos se encuentren articulados entre si por tejido conectivo denso, constituyendo las suturas ${ }^{16,22,36}$.

Las fontanelas conforman seis áreas de tejido conectivo denso correspondiente a la unión de dos o más suturas.

Se encuentran en la línea media la fontanela anterior (FA) (fonticulus anterior), y la (FP) (fonticulus posterior). Hacia los laterales del cráneo se localizan las antero-laterales (AL) (fonticulus anterolateralis) y las postero-laterales (PL) (fonticulus mastoideus) ${ }^{6,28,36}$.

Este trabajo tiene como objetivo describir la anatomía de las fontanelas, los hallazgos encontrados en disecciones cadavéricas y en pacientes pediátricos, y recordar las aplicaciones del conocimiento de las mismas.

Abreviaturas. AL: fontanela antero-lateral. DC: diámetro coronal. DS: diámetro sagital. FA: fontanela anterior. FP: fontanela posterior. PC: perímetro cefálico. PL: fontanela postero-lateral. SNC: sistema nervioso central. 


\section{Material y métodos}

Fueron utilizadas 7 cabezas cadavéricas de fetos, fijadas y conservadas con formol al 10\%, y 2 cráneos óseos secos obtenidos por osteotecnia $(\mathrm{n}=9)$. Asimismo se utilizaron 2 huesos frontales con su sutura metópica aún no consolidada.

En los preparados formolizados el abordaje del plano óseo y fontanelar se realizó con instrumental básico de disección, progresando desde la incisión en cuero cabelludo, hasta la diéresis de la gálea aponeurótica.

Fueron examinados 48 casos clínico-quirúrgicos en el Hospital de Niños "Sor María Ludovica" de la ciudad de La Plata, en los que la evaluación de la anatomía de las fontanelas fue relevante. Se analizaron dentro de los mismos: cirugías de craneosinostosis, punciones - drenajes transfontanelares y neuroecografías.

Todos los casos clínico-quirúrgicos fueron divididos en 5 grupos etáreos y se compararon sus promedios con el fin de evaluar la reducción del área fontanelar en el transcurso de los meses.

Los grupos fueron fraccionados en intervalos de 3 meses comenzando con el grupo A de 0 a 3 meses y finalizando con el grupo E de 12 meses en adelante.

Los promedios totales de los diámetros de las fontanelas fueron calculados con la siguiente fórmula, que combina tanto el diámetro coronal (DC) como el sagital (DS): $(\mathrm{DC}+\mathrm{DS}) / 2^{16}$.

\section{Resultados}

Se evidenció la estructura, límites y línea de inserción de las fontanelas impares y mediales, representadas por FA y la FP, y de las fontanelas pares y laterales, constituidas por la AL y la PL. Además se observó la diferencia en la superficie correspondiente a cada una de ellas y su relación topográfica con el endocráneo subyacente.

En las 9 preparaciones cadavéricas las edades aproximadamente variaron entre los 6 meses de gestación y el primer año postparto, los perímetros cefálicos (PC) de 21 a $36.5 \mathrm{~cm}$ (promedio $27.29 \mathrm{~cm}$ ), mientras que para sus FA los DC variaron entre 16 y $35 \mathrm{~mm}$ (promedio 25,89 $\mathrm{mm}$ ), mientras que los DS lo hicieron entre 22 y $45 \mathrm{~mm}$ (promedio $30 \mathrm{~mm}$ ), dando una relación DS/DC de 1.16.

Las mediciones de los 48 casos clínico-quirúrgicos

Tabla 1

\begin{tabular}{ccccc}
\hline & Edad & $\begin{array}{c}\text { Perímetro } \\
\text { cefálico }\end{array}$ & $\begin{array}{c}\text { FA } \\
\text { Sagital }\end{array}$ & $\begin{array}{c}\text { FA } \\
\text { Coronal }\end{array}$ \\
\hline Promedios & 4.72 & 39.88 & 31.42 & 27.92 \\
& meses & $\mathrm{cm}$ & $\mathrm{mm}$ & $\mathrm{mm}$ \\
\hline $\begin{array}{l}\text { Desvío } \\
\text { Stand. }\end{array}$ & 3.82 & 5.27 & 16.18 & 14.44 \\
\hline
\end{tabular}

arrojaron los resultados expuestos en la Tabla 1.

El intervalo de edad fue entre los 15 días de vida $\mathrm{y}$ los 17 meses, mientras que la relación $\mathrm{DS} / \mathrm{DC}$ fue de $1,12^{23,25}$.

La fontanela AL se encuentra en la confluencia de los huesos frontal, parietal, temporal y esfenoides (ala mayor), mientras que la PL se ubica en la intersección de las suturas témporo-parietal y lambdoidea. Ambas poseen una aplicación clínico-quirúrgica reducida, a excepción de $\mathrm{su}$ trascendente función fisiológica, eventual utilización como ventana ecográfica, y la presencia de patologías de baja frecuencia ${ }^{15,18}$ por lo que no fueron consideradas en este trabajo.

\section{Fontanela anterior (FA)}

Es la fontanela de mayor superficie y, por consiguiente, de mayor utilidad médica ${ }^{16}$.

Posee forma romboidal y se encuentra ubicada en la intersección de las suturas coronal, sagital y metópica. Esta última tiende a desaparecer hacia los 2 años de edad $^{6,16,28,36}$.

Sus medidas en el primer día de vida varían entre 6 y 36 $\mathrm{mm}$, media $21 \mathrm{~mm}^{16}$.

Generalmente cierra a los 13 - 14 meses (variabilidad entre 4 y 26 meses $)^{10}$.

En nuestra casuística, los diferentes grupos mostraron los valores expresados en las Tablas 2 y 3 .

La FA es la fontanela utilizada con mayor frecuencia

Tabla 2

\begin{tabular}{ccccc}
\hline Grupos & $\begin{array}{c}\text { Edad } \\
(\text { meses })\end{array}$ & $\begin{array}{c}\text { PC } \\
(\mathrm{cm})\end{array}$ & $\begin{array}{c}\text { FA DS } \\
(\mathrm{mm})\end{array}$ & $\begin{array}{c}\text { FA DC } \\
(\mathrm{mm})\end{array}$ \\
\hline Grupo A $(\mathbf{n = 2 0})$ & $0-2$ & 37.14 & 40.60 & 33.50 \\
\hline Grupo B $(\mathbf{n = 1 1 )}$ & $3-5$ & 39.08 & 27.55 & 27.09 \\
\hline Grupo C $(\mathbf{n = 8})$ & $6-8$ & 42.00 & 28.00 & 23.38 \\
\hline Grupo D $(\mathbf{n = 7})$ & $9-11$ & 44.93 & 19.14 & 21.14 \\
Grupo E $(\mathbf{n = 2})$ & 12 o más & 45.50 & 17.50 & 18.50 \\
\hline
\end{tabular}

Tabla 3

\begin{tabular}{ccc}
\hline Grupos & (DS+DC)/2 & $\begin{array}{c}\text { PC/ } \\
(D S+D C) / 2\end{array}$ \\
\hline Grupo A $(n=20)$ & 37,05 & 10,02 \\
\hline Grupo B $(n=11)$ & 27,32 & 14,30 \\
Grupo C $(n=8)$ & 25,69 & 16,35 \\
\hline Grupo D $(n=7)$ & 20,14 & 22,31 \\
Grupo E $(n=2)$ & 18,00 & 25,28 \\
\hline
\end{tabular}


para la realización de neuroecografías ${ }^{8,21,32,33,35}$, no sólo por ser la de mayor tamaño, sino por ser la de mayor latencia hasta desaparecer.

Relación endocraneal - Ventana acústica:

Fosa supratentorial.

Ventrículos laterales.

Lóbulos frontal y temporal.

\section{Fontanela posterior (FP)}

De conformación triangular, se encuentra en la concurrencia de las suturas sagital y lambdoidea ${ }^{6,28,36}$.

Mide luego del nacimiento aproximadamente de 5 a 7 $\mathrm{mm}^{16}$.

Generalmente se cierra alrededor de los 2 o 3 meses de vida $^{8,16}$.

Aventaja considerablemente a la FA al momento de evaluar por ultrasonografía a las estructuras más posteriores supra e infratentoriales ${ }^{7,18,35}$. Asimismo, eleva la posibilidad de detectar patologías respecto a la utilización aislada de la FA como ventana acústica ${ }^{7,8,18}$.

\section{Relación endocraneal - Ventana acústica:}

Fosa Supratentorial.

Ventrículos Laterales: cuerpo, asta occipital, asta temporal, plexos coroideos.

Lóbulos Occipital y Parietal.

\section{Discusión}

Las fontanelas están constituidas por tres capas: una externa, el periostio, que se continúa con el de los huesos adyacentes; una media, de tejido conectivo, conectada a los huesos que la rodean (capa que se osificará); y una interna, la duramadre ${ }^{36}$.

Clásicamente se describen seis fontanelas (Figura 1). Se encuentran en el plano sagital la FA, mayor o bregmática (Figura 2) y la FP, menor o lámbdica (Figura 3). En las caras laterales del cráneo y con menor superficie, se localizan las AL, esfenoidales o ptéricas; y las PL, mastoideas o astéricas (Figura 4) ${ }^{6,28,36}$.

Asimismo es factible la presencia de fontanelas accesorias, como es el caso de la fontanela sagital (o fontanela de Gerdy), localizada en la sutura homónima a nivel del obelion, que suele relacionarse con síndrome de Down o hipotiroidismo ${ }^{16,33,36}$. Otras fontanelas supernumerarias son la nasofrontal, ubicada entre las dos mitades del frontal y los huesos propios de la nariz (en el adulto se osifica en un hueso supranasal); la fontanela metópica, localizada en la sutura homónima (Figura 5) (puede osificarse en un hueso metópico, supernumerario); y la fontanela cerebelosa, situada por detrás del agujero magno ${ }^{16,33,36}$.

En la evolución normal del desarrollo y crecimiento craneal, el perímetro cefálico aumenta, las suturas se con-

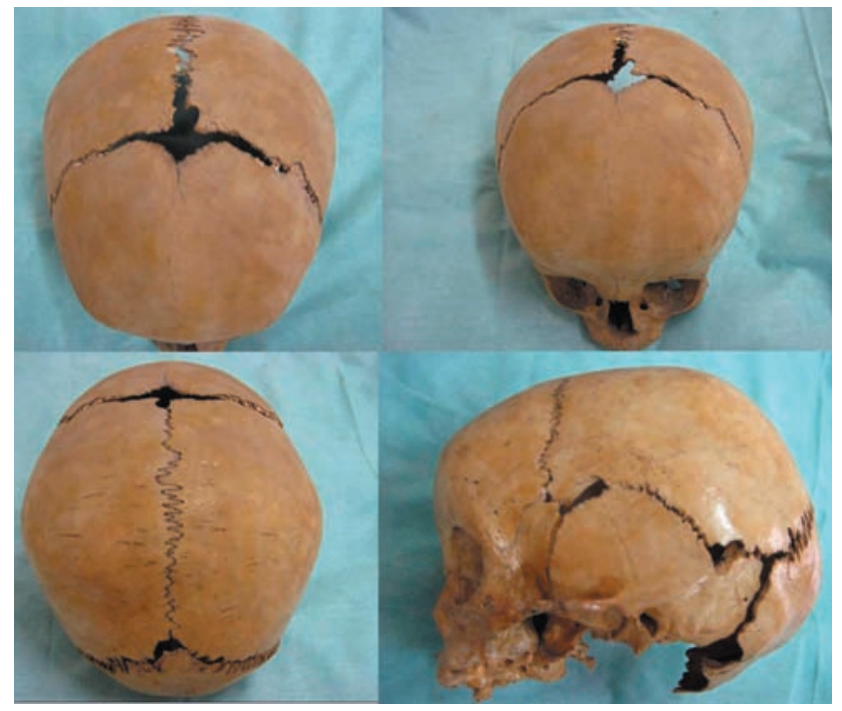

Figura 1. Diferentes vistas de las fontanelas en cráneo desarticulado y reconstruido.

solidan y las fontanelas se extinguen para transformarse en puntos de relevancia craneométrica, donde convergen las distintas suturas ${ }^{6,36}$.

Hemos evidenciado en nuestra casuística que si bien la superficie fontanelar tiende a disminuir con el transcurso de los meses, lo hace a un ritmo más lento que en lo descrito en la bibliografía clásica, hecho que atribuimos a haber realizado la mayoría de las mediciones en pacientes con patología subyacente, en especial, neuroquirúrgica.

También resaltamos un leve predominio del DS sobre el DC en la fontanela anterior si se analizan los promedios generales, existiendo para casos individuales la equidad entre ambas medidas o incluso su inversa.

El conocimiento de la anatomía de las fontanelas es relevante para comprender y reconocer la aparición de patologías del cráneo óseo, del sistema nervioso central (SNC), o de otro sistema que se reflejen sobre las mismas.

Sus aplicaciones tienen interés en diferentes aspectos:

\section{Semiología}

La presión intracraneal (PIC) es el resultado de un equilibro de presiones dado por los diferentes sistemas que contribuyen a su génesis. Sus variaciones, sean positivas o negativas, repercuten de manera disímil en el adulto y en el neonato.

Por ello, ante una elevación de la misma en un cráneo con suturas no consolidadas, se produce una distorsión de su conformación normal, llevando así a un abombamiento de todas las fontanelas y un incremento del perímetro cefálico (v.gr. meningoencefalitis, hidrocefalia, tumores, traumatismos $)^{16}$.

Del mismo modo, una disminución de la PIC produce una depresión de las fontanelas, lo que orienta, por ejem- 


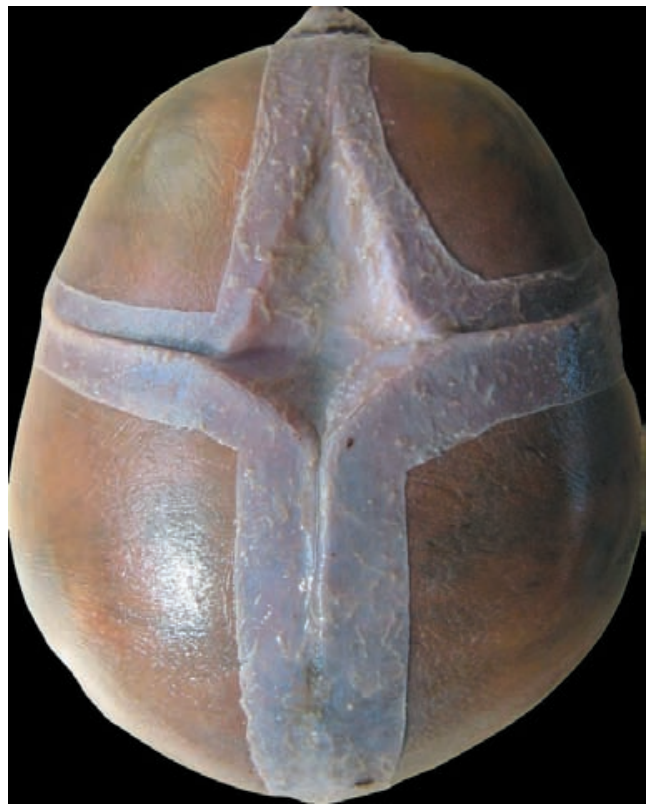

Figura 2. Vista craneal superior: fontanela anterior.

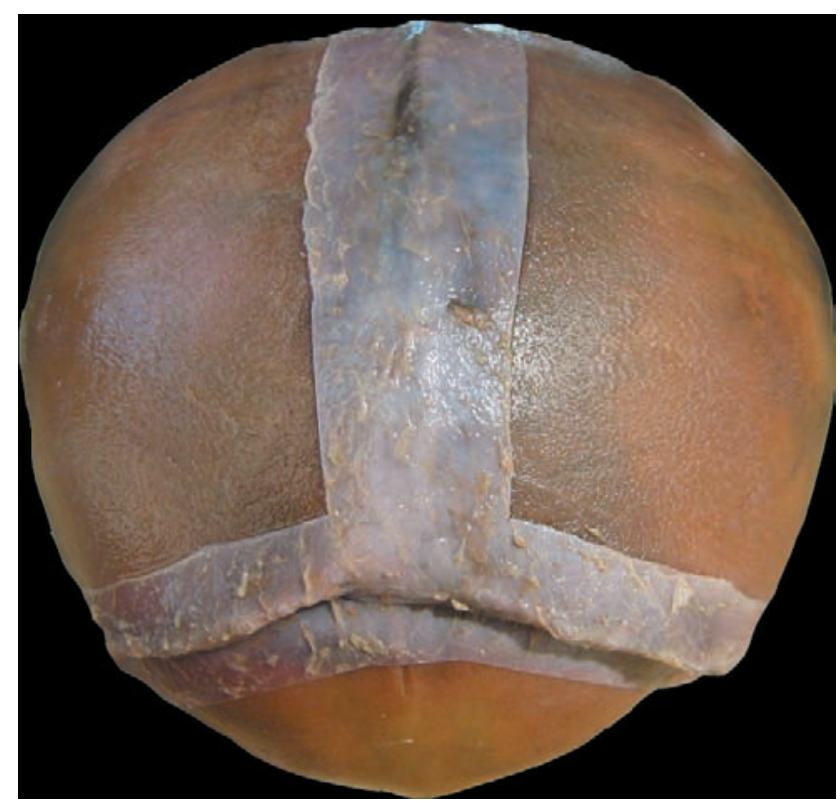

Figura 3. Vista craneal posterior: fontanela posterior.

plo, hacia una deshidratación moderada o grave ${ }^{16}$.

Como se ha dicho, la FA cierra escaso tiempo después del año de vida, su persistencia es sinónimo de patología asociada como procesos que eleven la PIC, acondroplasia, osteogénesis imperfecta, hipotiroidismo, déficit de vitamina $\mathrm{D}$, displasia cleidocraneal, pyknodisostosis, o cromosomopatías como el síndrome de Down u otros menos frecuentes (trisomías 13 o 18, Russell - Silver, Rubinstein

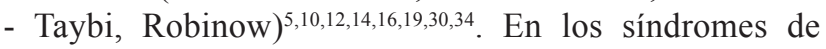
displasia cleidocraneal y Frank-ter Haar se observa una FA de diámetros amplios $5,9,12,19,30,34$.

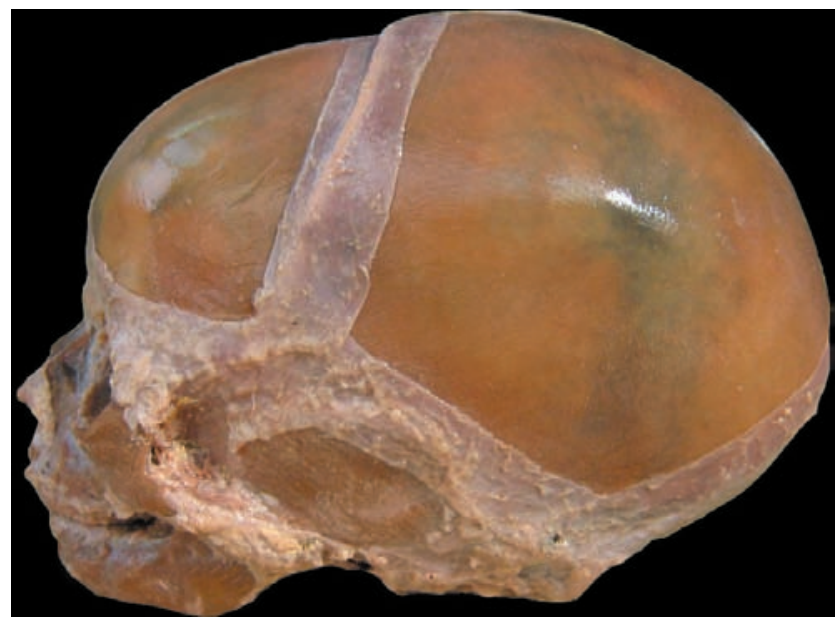

Figura 4. Vista craneal lateral: fontanelas antero y posterolateral.

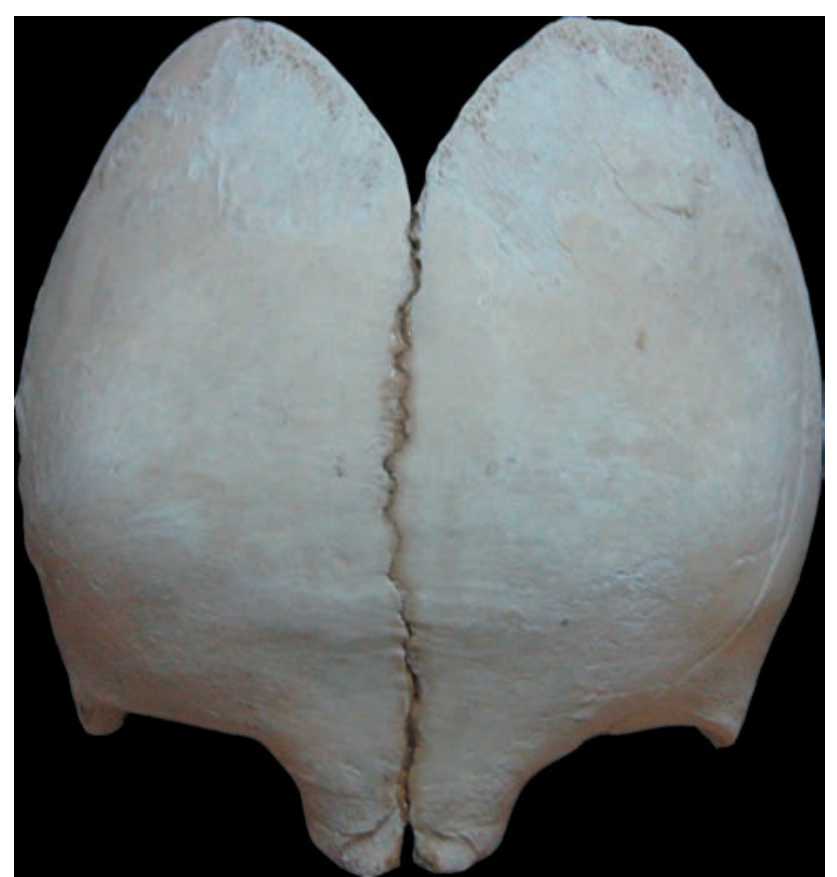

Figura 5. Vista anterior del hueso frontal: sutura metópica $y$ parte anterior de fontanela anterior.

La craneosinostosis es el cierre prematuro de una o más suturas de los huesos del cráneo, y se relaciona ocasionalmente con fontanelas pequeñas u oclusión prematura de las mismas ${ }^{12,16,37}$.

$\mathrm{Si}$ bien son raras las tumoraciones que tienen asiento sobre la FA, pueden citarse a los quistes dermoides, meningo o encefaloceles, quistes sebáceos, cefalohematomas, linfangiomas o hemangiomas ${ }^{1,2}$. A nivel de la FP una tumoración puede corresponder a un cefalocele atrésico, y en el caso de las fontanelas laterales (ptérica y astérica) a un meningoencefalocele temporal lateral espontáneo ${ }^{15,26}$. 


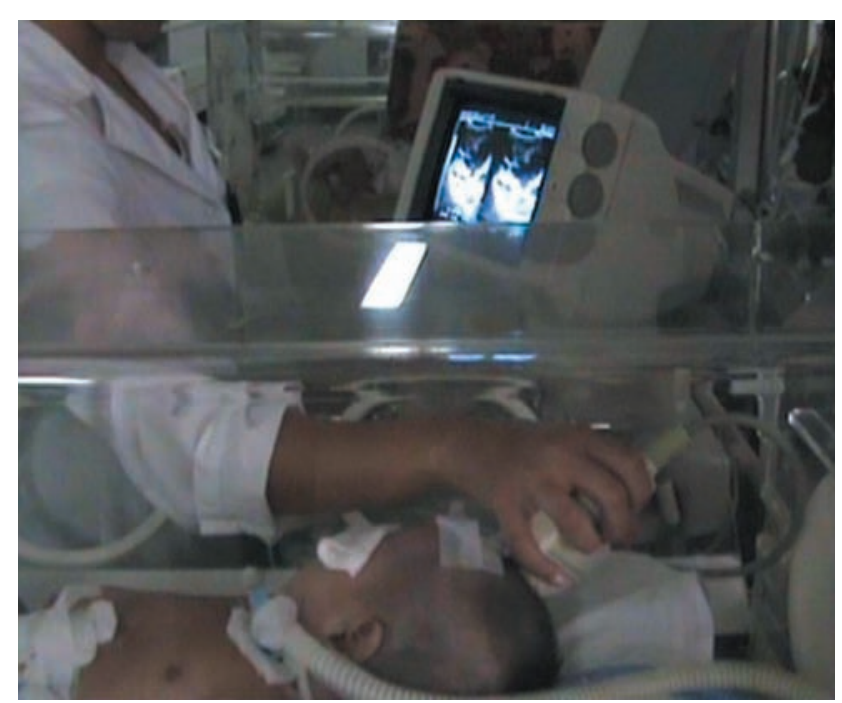

Figura 6. Realización de ecografía transfontanelar dentro de la incubadora.

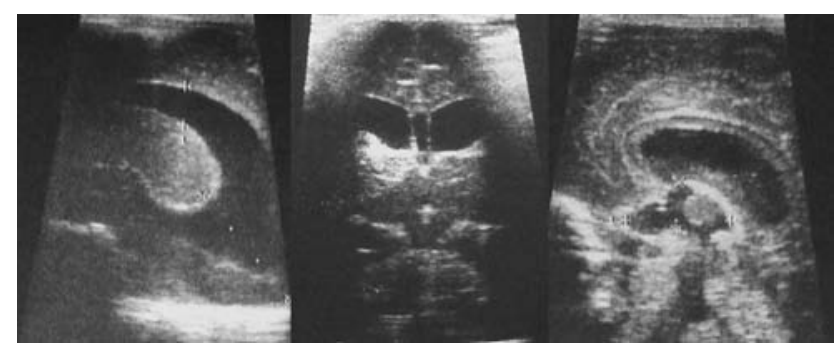

Figura 7. Neuroecografía vía transfontanelar. Cortes sagitales y coronales donde se muestra una dilatación ventricular por hidrocefalia.

\section{Diagnóstico por imágenes. Neuroecografía}

En el paciente adulto, debido a la continuidad ósea del cráneo, las ecografías del $\mathrm{SNC}$ se realizan cuando éste ha sido abordado quirúrgicamente ${ }^{20}$.

La ecografía transfontanelar o neuroecografía es un método de diagnóstico por imágenes de gran utilidad al momento de estudiar el SNC, en todo paciente pediátrico que conserve un adecuado tamaño de sus fontanelas (Figura 6). Permite obtener tanto cortes coronales, como sagitales y parasagitales a través de la FA ${ }^{32,33,35}$ (Figura 7) y realizar mediciones ${ }^{4}$.

La evaluación ecográfica de las fontanelas y suturas craneales resulta de utilidad en el diagnóstico prenatal de diversas patologías ${ }^{11}$.

Todas las imágenes son obtenidas en tiempo real, por lo que puede visualizarse incluso los latidos arteriales ${ }^{33}$. La disponibilidad de neuroecografía transfontanelar intraoperatoria resulta de utilidad para identificar lesiones encefálicas profundas ${ }^{20}$.

Puede además utilizarse la técnica de Doppler color, al momento de evaluar los vasos sanguíneos intracraneales,

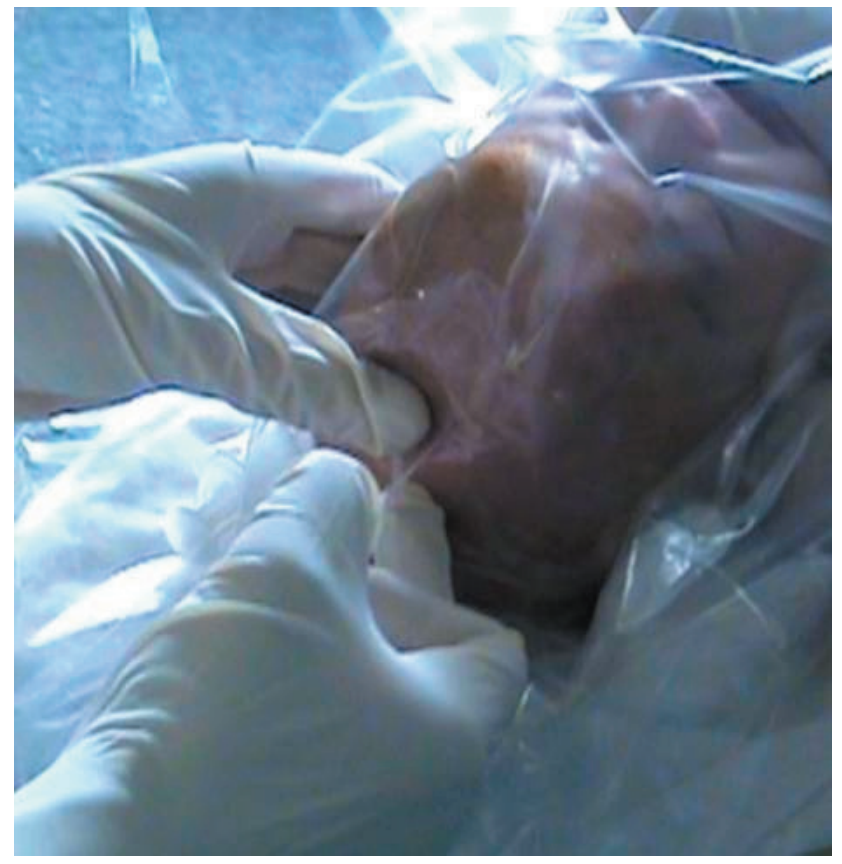

Figura 8. Técnica de punción transfontanelar (FA).

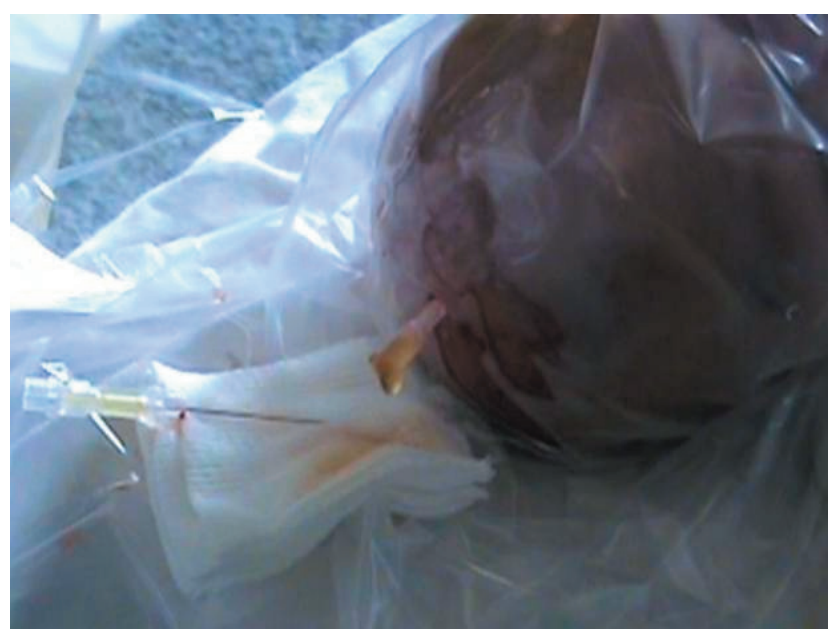

Figura 9. Líquido cefalorraquídeo purulento obtenido por punción transfontanelar.

índices de resistencia de las arterias cerebrales u otras estructuras (v.gr. detección de aneurisma de ampolla de Galeno, evaluación perioperatoria de la compliance intracraneana en una craneosinostosis) $)^{3,8,21,37,38}$. También resulta de utilidad en el período neonatal para el diagnóstico de: desórdenes metabólicos (v.gr. desórdenes en la fosforilación oxidativa, desórdenes en la biogénesis peroxisómica), y de hemorragias intracraneales y sus consecuencias $^{17,29}$.

La evaluación ecográfica de las fontanelas puede integrarse al examen del resto de las estructuras óseas craneanas con técnicas tridimensionales o estereoscópicas ${ }^{24}$. 
Si bien la FA es la más usada, otras fontanelas, e incluso la escama del hueso temporal ${ }^{8,13}$ y el foramen magnum ${ }^{18}$, pueden ser utilizadas como ventana acústica, sobre todo al momento de evaluar la fosa posterior, cuya visualización resulta dificultosa con el transductor ecográfico ubicado en la $\mathrm{FA}^{8,35}$.

Para ubicar el transductor ecográfico en las fontanelas posterior y postero-lateral se prefiere el decúbito lateral, mientras que para las realizadas en la FA puede adoptarse casi cualquier decúbito ${ }^{8}$, hecho a destacar en pacientes con movilidad limitada.

Además de obtener imágenes del SNC, la neuroecografía permite guiar procedimientos terapéuticos invasivos en tiempo real, y realizar monitoreo intraoperatorio (v.gr. colocación de un shunt ventricular, punciones de cavidades quísticas de situación profunda) $)^{3,20}$.

La ecografía transfontanelar mantiene sustanciosas ventajas frente a otros procedimientos para la obtención de neuroimágenes: $:^{3,32,33}$

- Estudio en tiempo real.

- Equipo portátil: puede realizarse aún con el paciente dentro de la incubadora.

- No tiene exposición a radiaciones.

- No necesita sedación o anestesia general.

- Bajo costo.

\section{Métodos invasivos. Punciones}

$\mathrm{Al}$ carecer la FA de estructura ósea, es posible realizar a través de la misma procedimientos diagnósticos y terapéuticos invasivos, además de los procedimientos diagnósticos no invasivos considerados anteriormente.

Pueden efectuarse punciones transfontanelares con fines diagnósticos (v.gr. meningoencefalitis (Figuras 8 y 9) o terapéuticos (v.gr. drenaje ventricular de líquido cefalorraquídeo $)^{27,31}$.

\section{Conclusión}

Fisiológicamente es destacable su función al momento del parto, ya que junto a las suturas posibilita el amoldamiento ${ }^{1}$.

Debido a las características estructurales y topográficas de las fontanelas, éstas se comportan como una verdadera ventana hacia el endocráneo, permitiendo aplicaciones clínico-semiológicas, diagnósticas (ecografía transfontanelar) y terapéuticas (punciones, drenaje ventricular y de abscesos).

El conocimiento de la anatomía de las fontanelas y su relación con las diferentes estructuras endocraneales, permite una mejor comprensión de patologías regionales o a distancia que repercuten en ellas, así como también sus utilidades en las diferentes aplicaciones médicas y quirúrgicas.

\section{Bibliografía}

1. Agrawal, A., Pratap, A., Sinha, A.K.: Epidermoid cyst of anterior fontanelle with clear contents. Surgical Neurology 2007; 68: 313-316.

2. Aslan, Ö., Özveren, F., Kotil, K.: Congenital Dermoid Cyst of the Anterior Fontanelle in Turkish Children. Neurol Med Chir (Tokyo) 2004; 44: 150-152.

3. Babcock, D.S.: Sonography of the Brain in Infants: Role in Evaluating Neurologic Abnormalities. AJR 1995; 165: 417423.

4. Barkovich, A.J.: The Encephalopathic Neonate: Choosing the Proper Imaging Technique. AJNR 1997; 18: 18161820 .

5. Bathi, R.J., Masur, V.N.: Pyknodysostosis - a report of two cases with a brief review of the literature. Int. J. Oral Maxillofac. Surg. 2000; 29: 439-442.

6. Bouchet, A., Cuilleret, J.: Cráneo del Recién Nacido. Fontanelas. En: Sistema Nervioso Central. Anatomía Descriptiva, Topográfica y Funcional. Buenos Aires; Ed. Panamericana, 1978; p. 30.

7. Correa, F., Enrríquez, G., Rosselló, J., et al.: Posterior Fontanelle Sonography: An Acoustic Window into the Neonatal Brain. Am J Neuroradiol 2004; 25:1274-1282.

8. Disalvo, D.N.: A New View of the Neonatal Brain: Clinical Utility of Supplemental Neurologic US Imaging Windows. RadioGraphics 2001; 21: 943-955.

9. Dundar, M., Saatci, C., Tasdemir, S.: Frank-ter Haar syndrome with unusual clinical features. European Journal of Medical Genetics (in press).

10. Gaetano, H., Gaetano, J.S.: Persistent open anterior fontanelle in a healthy 32-month-old boy. JAOA 2002; 102: 500-501.

11. Ginath, S., Debby, A., Malinger, G.: Demonstration of cranial sutures and fontanelles at 15 to 16 weeks of gestation: a comparison between two-dimensional and three-dimensional ultrasonography. Prenat Diagn 2004; 24: 812-815.

12. Glass, R.B.J., Fernbach, S.K., Norton K.I.: The Infant Skull: A Vault of Information. RadioGraphics 2004; 24: $507-$ 522.

13. Hirsch, W., Hiebsch, W., Teichler, H.: Transcranial Doppler Sonography in Children: Review of a Seven-year Experience. Clinical Radiology 2002; 57: 492-497.

14. Holick, M.F., Lim, R., Dighe, A.S.: Case 3-2009: A 9Month-Old Boy. N Engl J Med 2009; 360: 398-407.

15. Horky, J.K., Chaloupka, J.C., Putman, C.M.: Occult Spontaneous Lateral Temporal Meningo-encephalocele: MR Findings of a Rare Developmental Anomaly. AJNR 1997; 18: 744-746.

16. Kiesler, J., Ricer, R.: The Abnormal Fontanel. Am Fam Physician 2003; 67: 2547-2552.

17. Leijser, L.M., de Vries, L.S., Rutherford, M.A., et al.: Cranial Ultrasound in Metabolic Disorders Presenting in the 
Neonatal Period: Characteristic Features and Comparison with MR Imaging. Am J Neuroradiol 2007; 28: 1223-1231.

18. Luna, J., Goldstein, R.: Sonographic Visualization of Neonatal Posterior Fossa Abnormalities Through the Posterolateral Fontanelle. AJR 2000; 174: 561-567.

19. Mata Zubillaga, D., Lapeña López de Armentia, S.: Disostosis cleidocraneal. Revisión de once casos en cinco generaciones. An Pediatr (Barc). 2008; 69: 162-166.

20. Merritt, C.R., Coulon, R., Connolly, E.: Intraoperative Neurosurgical Ultrasound: Transdural and Transfontanelle Applications. Radiology 1983; 148: 513-517.

21. Mitchell, D.G., Merton, D., Needleman, L., et al.: Neonatal Brain: Color Doppler Imaging Part I. Technique and Vascular Anatomy. Radiology 1988; 167: 303-306.

22. Moore, K.L., Persaud, T.V.N.: Sistema esquelético. Desarrollo del cráneo. En. Embriología Clínica. México; Ed. Interamericana, McGraw-Hill, 1995; pp. 386-388.

23. Mota, M., Melo, A., Burak, C.: Antropometria Craniana de Recém-Nascidos Normais. Arq Neuropsiquiatr 2004; 62(3-A): 626-629.

24. Nelson, T.R., Ji, E.K., Lee, J.H.: Stereoscopic Evaluation of Fetal Bony Structures. J Ultrasound Med 2008; 27: 15-24.

25. Oliveira, H.A., da Paixão, A.C., de Oliveira R. Paixão, M.: Estudo Antropométrico do Craânio de Recém-Nascidos Normais em Sergipe. Arq Neuropsiquiatr 2007; 65(3-B): 896899.

26. Patterson, R.J., Egelhoff, J.C., Crone, K.R.: Atretic Parietal Cephaloceles Revisited: An Enlarging Clinical and Imaging Spectrum? Am J Neuroradiol 1998; 19: 791-795.

27. Ros-López, B., Jaramillo-Dallimonti, A.M., De Miguel-Pueyo, L.S., et al.: Hemorragia intraventricular del prematuro e hidrocefalia post-hemorrágica. Propuesta de un protocolo de manejo basado en la derivación ventrículoperitoneal precoz. Neurocirugía 2009; 20: 15-24.

28. Rouvière, H., Delmas, A.: Desarrollo del Cráneo. Fontanelas. En: Anatomía Humana. Descriptiva, Topográfica y Funcional. Tomo 1, Esqueleto de la Cabeza y Cuello. Barcelona; Ed. Masson, 2005; pp. 89-90.
29. Sauerbrei, E.E., Cooperberg, P.L., Harrison, P.E.: Realtime trans-fontanelle ultrasonic demonstration of neonatal intracranial pathology. RadioGraphics 1981; 1: 15-28.

30. Shen, W.: A Case of Cleidocranial Dysplasia Confirmed by 3D CT of the Cranium. AJNR 2000; 21: 609 (letter).

31. Shooman, D., Portess, H., Sparrow, O.: A review of the current treatment methods for posthaemorrhagic hydrocephalus of infants. Cerebrospinal Fluid Research 2009; 6: 1.

32. Shuman, W.P., Rogers, J.V., Mack, L.A.: Real-Time Sonographic Sector Scanning of the Neonatal Cranium: Technique and Normal Anatomy. AJR 1981; 137: 821-828.

33. Slovis, T., Kuhns, L.: Real-Time Sonography of the Brain through the Anterior Fontanelle. AJR 1981; 136: 277286.

34. Soto, E., Richani, K., Gonçalves, L.F., et al.: Threedimensional ultrasound in the prenatal diagnosis of cleidocranial dysplasia associated with B-cell immunodeficiency. Ultrasound Obstet Gynecol 2006; 27: 574-579.

35. Steggerda, S.J., Leijser, L.M., Walther, F.J.: Neonatal cranial ultrasonography: How to optimize its performance. Early Human Development 2009; 85: 93-99.

36. Testut, L., Latarjet, A.: Cabeza ósea. Desarrollo general del cráneo. En: Tratado de Anatomía Humana. Barcelona; Ed. Salvat Editores S.A., 1954; pp. 210-213.

37. Westra, S.J., Stotland, M.A., Lazareff, J.: Perioperative Transcranial Doppler US to Evaluate Intracranial Compliance in Young Children Undergoing Craniosynostosis Repair Surgery. Radiology 2001; 218: 816-823.

38. White, B.D., Bis, K.G., Cacciareii, A.A.: Ultrasound Case of the Day. Radio-Graphics 1992; 12: 396-400.

Bustamante, J.L.; Miquelini, L.A.; D’Agustini, M.; Fontana, A.M.: Anatomía aplicada de las fontanelas. Neurocirugía 2010; 21: 253-259.

Correspondencia: Jorge Luis Bustamante. Cátedra de Anatomía "C". Facultad de Ciencias Médicas. Universidad Nacional de La Plata. La Plata, Buenos Aires, Argentina. jorgeluisbustamante@hotmail.com 